\title{
GEMINI SPECTROSCOPY OF THE SHORT-HARD GAMMA-RAY BURST GRB 130603B AFTERGLOW AND HOST GALAXY
}

\author{
A. Cucchiara ${ }^{1}$, J. X. Prochaska ${ }^{1}$, D. Perley ${ }^{2,7}$, S. B. Cenko ${ }^{3}$, J. Werk ${ }^{1}$, A. Cardwell $^{4}$, \\ J. TURNER ${ }^{4}$, Y. CAO ${ }^{2}$, J. S. BLOOM ${ }^{5}$, AND B. E. СOBB 6 \\ ${ }^{1}$ Department of Astronomy and Astrophysics, UCO/Lick Observatory, University of California, \\ 1156 High Street, Santa Cruz, CA 95064, USA; acucchia@ucolick.org \\ 2 Department of Astronomy, California Institute of Technology, MC 249-17, 1200 East California Blvd, Pasadena, CA 91125, USA \\ ${ }^{3}$ Astrophysics Science Division, NASA Goddard Space Flight Center, Greenbelt, MD, USA \\ ${ }^{4}$ Gemini South Observatory, AURA, Casilla 603, La Serena, Chile \\ ${ }^{5}$ Department of Astronomy, University of California, Berkeley, CA 94720-3411, USA \\ 6 The George Washington University, Washington, DC, USA \\ Received 2013 June 9; accepted 2013 August 25; published 2013 October 18
}

\begin{abstract}
We present early optical photometry and spectroscopy of the afterglow and host galaxy of the bright short-duration gamma-ray burst GRB 130603B discovered by the Swift satellite. Using our Target of Opportunity program on the Gemini South telescope, our prompt optical spectra reveal a strong trace from the afterglow superimposed on continuum and emission lines from the $z=0.3568 \pm 0.0005$ host galaxy. The combination of a relatively bright optical afterglow $\left(r^{\prime}=21.52\right.$ at $\Delta t=8.4 \mathrm{hr}$ ), together with an observed offset of 0.9 from the host nucleus ( $4.8 \mathrm{kpc}$ projected distance at $z=0.3568$ ), allow us to extract a relatively clean spectrum dominated by afterglow light. Furthermore, the spatially resolved spectrum allows us to constrain the properties of the explosion site directly, and compare these with the host galaxy nucleus, as well as other short-duration GRB host galaxies. We find that while the host is a relatively luminous $\left(L \approx 0.8 L_{B}^{*}\right)$, star-forming $\left(\mathrm{SFR}=1.84 \mathrm{M}_{\odot} \mathrm{yr}^{-1}\right.$ ) galaxy with almost solar metallicity, the spectrum of the afterglow exhibits weak Ca II absorption features but negligible emission features. The explosion site therefore lacks evidence of recent star formation, consistent with the relatively long delay time distribution expected in a compact binary merger scenario. The star formation rate (SFR; both in an absolute sense and normalized to the luminosity) and metallicity of the host are both consistent with the known sample of short-duration GRB hosts and with recent results which suggest GRB 130603B emission to be the product of the decay of radioactive species produced during the merging process of a neutron-star-neutron-star binary ("kilonova"). Ultimately, the discovery of more events similar to GRB $130603 \mathrm{~B}$ and their rapid follow-up from $8 \mathrm{~m}$ class telescopes will open new opportunities for our understanding of the final stages of compact-objects binary systems and provide crucial information (redshift, metallicity, and chemical content of their explosion site) to characterize the environment of one of the most promising gravitational wave sources.
\end{abstract}

Key words: gamma-ray burst: individual (GRB 130603B) - techniques: imaging spectroscopy

Online-only material: color figures

\section{INTRODUCTION}

Short gamma-ray bursts (SGRBs) are historically identified based on the duration of their gamma-ray emission $\left(T_{90} \lesssim 2 \mathrm{~s}^{8}\right)$ and their hard spectrum (e.g., Mazets et al. 1981; Norris et al. 1984; Kouveliotou et al. 1993). Recently, based on a statistical approach, several attempts have been made to improve this classification (Ř́pa et al. 2009; Veres et al. 2010; Bromberg et al. 2013). Short-GRBs also differ from the "long" GRBs class for their redshift distribution and, likely, their progenitors (see, for example, Li \& Paczyński 1998; O'Shaughnessy et al. 2008; Zhang et al. 2009; Kann et al. 2011, and references therein).

The optical afterglows of SGRBs are on average two orders of magnitude less optically luminous than their long duration counterparts (Kann et al. 2011), making broadband followup, and optical spectroscopy in particular, quite challenging. Nevertheless, the Swift satellite (Gehrels et al. 2004) has enabled the localization of a modest sample in X-rays and a smaller set have been detected in optical/near-IR passbands (e.g., Fox et al. 2005; Bloom et al. 2006; Hjorth et al. 2005; Berger 2007; Nakar 2007; Fong et al. 2013a).

\footnotetext{
7 Hubble Fellow.

8 Time over which a burst emits from $5 \%$ of its total measured counts to $95 \%$.
}

In contrast, long GRBs $\left(T_{90} \gtrsim 2 \mathrm{~s}\right)$ present brighter afterglows allowing accurate localization and spectroscopic follow-up hours after the events occur. Robotic facilities and Target of Opportunity $(\mathrm{ToO})$ programs have provided a plethora of photometric and spectroscopic data in support of theoretical models of long GRB progenitors and the host galaxies they live in. These data first established conclusively the extragalactic nature of the events (Metzger et al. 1997) and, eventually, analysis of the Ly $\alpha$ forest for high- $z$ events (e.g., Salvaterra et al. 2009; Tanvir et al. 2009; Cucchiara et al. 2011) and fine-structure transitions at lower redshift provided unambiguous physical associations to their hosts. Optically bright long GRBs seem exclusively hosted by star-forming galaxies with high specific star-formation and sub- $\mathrm{L}^{*}$ luminosities, indicating massive stars as likely progenitors of long GRBs (see Levesque 2013, and reference therein), while "dark" GRBs (Jakobsson et al. 2004) seem to be harbored, on average, in more massive and highly star-forming galaxies $\left(3 \times 10^{10} M_{\odot}\right.$ at $z \approx 2$, Perley et al. 2013).

Thanks to a concerted community effort, of the $\sim 70$ short GRBs detected by Swift $\sim 1 / 3$ have been localized to within a few arcseconds accuracy, and, with a a posteriori probabilistic arguments, have been securely associated with nearby galaxies (see, e.g., Fong et al. 2013a; Fong \& Berger 2013). The number 
of these events, however, is still in the few dozens (Fong et al. 2013a). The few well-observed SGRBs have been associated primarily to a population of galaxies very similar to field galaxies at similar redshifts with moderate to negligible star formation rate (SFR), lending credence to the idea that at least some SGRBs explode with delay times $\gtrsim 1$ Gyr consistent with the progenitor model of compact mergers (e.g., neutron star binaries, or neutron star-black hole; Lee et al. 2005, 2010; Hjorth et al. 2005). Recently, using deep observations from space and from the ground it has been possible to quantify the relative fraction short-duration GRB hosts: $20 \%-40 \%$ earlyand 60\%-80\% late-type galaxies (Berger 2010; Fong et al. 2013a).

From the afterglow perspective, there has yet to be a bona fide short duration GRB afterglow for which we have measured a redshift from absorption features in the optical spectrum. Among these it is important to note the long GRB 090426, for which the duration of the prompt emission $\left(t_{90}=1.2 \mathrm{~s}\right)$ and the properties of the host made its classification uncertain (Antonelli et al. 2009; Levesque et al. 2010). Another debated short GRB for which an afterglow+host galaxy spectrum has been obtained is GRB 051221A (Soderberg et al. 2006): also in this case the non-collapsar nature of this event has been recently questioned based on probabilistic arguments and an accurate analysis of the instrumental biases which may lead one to mistakenly associate collapsar events like this one to shortGRBs (Bromberg et al. 2013). Finally, GRB 100816A, despite having $t_{90} \approx 2 \mathrm{~s}$, has been associated with the SGRB class based on lag analysis (Norris et al. 2010) and its afterglow (or a combination of afterglow and host) has been spectroscopically observed (Tanvir et al. 2010; Gorosabel et al. 2010). The lack of a large sample of SGRB afterglow spectra has made it impossible to conduct analogous studies of the host galaxy ISM and circumburst environment, as are routinely achieved for long-duration events.

Finally, the lower rate of space-based localization (compared to the long GRB class) and their faintness demand a very rapid response by large area telescopes to reach the quickly fading afterglows and obtain similar high-quality data for a larger sample of SGRBs. Such a collection will serve two main goals: (1) provide unambiguously, based, e.g., on absorptionline diagnostics like fine-structure transition, the redshift of these events and their association with an underlying host galaxy; (2) directly allow the characterization of the interstellar and circumstellar environment from 200 to $300 \mathrm{pc}$ up to the host halo (Chen et al. 2005; Prochaska et al. 2006; Berger et al. 2006; Vreeswijk et al. 2013) and, then, provide clues about the progenitor itself.

In this paper we present our prompt optical spectroscopic and photometric observations of the short GRB 130603B, obtained at the Gemini South telescope. Based on the optical and near infrared emission at late time ( $\sim 9$ days post-burst) derived by deep Hubble Space Telescope (HST) observations which allowed to clearly resolve the GRB-host complex, it has been proposed that this event might resemble the expected emission due to the decay of radioactive species produced and initially ejected during the merging process of a neutron star's binary system, referred to as a "kilonova" (Li \& Paczyński 1998; Metzger \& Berger 2012; Berger et al. 2013; Tanvir et al. 2013).

Thanks to our ToO program, we observed this event within the first day, when the afterglow dominated the host flux despite their small projected separation. This provides constraints on the event redshift and the properties of the GRB explosion site, in particular, in comparison to the overall SGRB host galaxy population.

The paper is divided as follows: in Section 2 we present our observing campaign; in Section 3 we present our spectral analysis, and in Section 4 we discuss our results and compare them with previous studies on SGRBs and their host galaxies. Finally, in Section 5 we present some implications on the nature of GRB 130603B and the possibilities offered by rapid response facilities for SGRB studies. Throughout the paper, we will use the standard cosmological parameters, $H_{0}=70 \mathrm{~km} \mathrm{~s}^{-1} \mathrm{Mpc}^{-1}$, $\Omega_{m}=0.27$, and $\Omega_{\Lambda}=0.73$.

\section{OBSERVATIONS AND DATA REDUCTION}

On 2013 June 3, at $T_{0}=15: 49: 14$ UT, the Swift satellite (Gehrels et al. 2004) triggered on GRB 130603B (Melandri et al. 2013). The onboard Burst Alert Telescope (BAT; Barthelmy et al. 2005) detected a single bright peak with a duration of $0.4 \mathrm{~s}$, placing this event unambiguously in the short-GRB category (Norris et al. 2013). After slewing to the source location, the X-ray Telescope (XRT; Burrows et al. 2005), began observing $59 \mathrm{~s}$ after the trigger, and detected a fading X-ray counterpart at $\alpha=11^{\mathrm{h}} 28^{\mathrm{m}} 48^{\mathrm{s}} .16, \delta=+17^{\circ} 04^{\prime} 18^{\prime \prime} .8$ (with an uncertainty of 2.'7; Evans et al. 2013). No optical counterpart was found in the UV/Optical Telescope Roming et al. (2005) prompt data (Melandri et al. 2013), while a counterpart was detected when more data became available (de Pasquale \& Melandri 2013).

At $T_{0}+5.8 \mathrm{hr}$, using the William Herschel Telescope, Levan et al. (2013) identified a point-like source inside the XRT error circle, which they determined to be the optical counterpart of the short GRB 130603B. This position lies in the outskirts of a galaxy present in the archival Sloan Digital Sky Survey (SDSS) DR9 (Ahn et al. 2012). Other subsequent follow-up observations were performed on GRB 130603B yielding a spectroscopic redshift for the afterglow and/or the host galaxy (Thoene et al. 2013; Xu et al. 2013; Sánchez-Ramírez et al. 2013; Foley et al. 2013; Cucchiara et al. 2013). The afterglow was also later detected at radio wavelengths (Fong et al. 2013b). Unfortunately, no observations to date have been providing detections of fine-structure lines, which are connected to the GRB radiation itself (leaving still some uncertainty on the actual GRB-host association).

Using our ToO program (GS-2013A-Q-31; PI Cucchiara) we performed a series of photometric observations with the GMOS camera (Hook et al. 2004) on Gemini South in the $g^{\prime}$, $r^{\prime}$, and $i^{\prime}$ filters for a total of 8x180 s exposures per band from $T=T_{0}+7.19 \mathrm{~h}$ to $T=T_{0}+9.1 \mathrm{~h}$. The data were analyzed using the standard GEMINI/GMOS data analysis packages within the IRAF ${ }^{9}$ environment. The afterglow is detected at a projected distance of $\approx 0.92 \pm 0$ '. 10 from the center of a bright, neighboring galaxy (see the false-color image in Figure 1). At the galaxy's redshift (see next section), this corresponds to $4.8( \pm 0.5) \mathrm{kpc}$ in projected distance.

Subsequently, we obtained a spectroscopic sequence with the same instrument: we obtained $2 \times 900$ s spectra, using the R400 grism with the $1^{\prime \prime}$ slit (resolution of about $5.5 \AA$ ) centered at $6000 \AA$, covering wavelengths $3900-8100 \AA$. We reduced the spectroscopic data with standard techniques, performing flat-fielding, wavelength calibration with $\mathrm{CuAr}$ lamp spectra, and cosmic ray rejection using the lacos_spec package (van

\footnotetext{
9 IRAF is distributed by the National Optical Astronomy Observatory, which is operated by the Association for Research in Astronomy, Inc., under cooperative agreement with the National Science Foundation.
} 

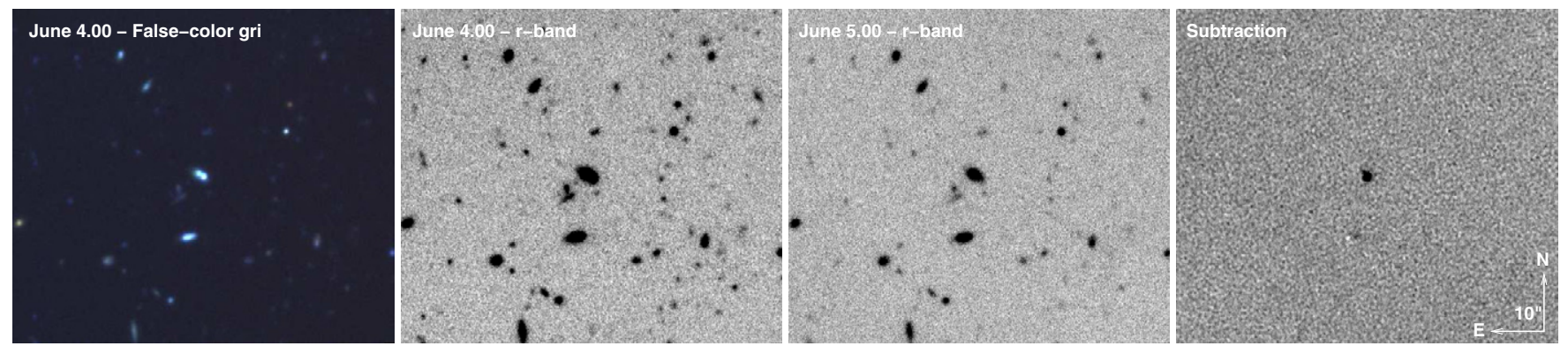

Figure 1. From left to right: composite false-color image of GRB 130603B and associated galaxy obtained the night of the discovery with Gemini/GMOS; $r^{\prime}$ band coadded image obtained the first night; $r^{\prime}$ band coadded image obtained the second night; host galaxy subtracted image of the GRB.

(A color version of this figure is available in the online journal.)

[OII] 3727
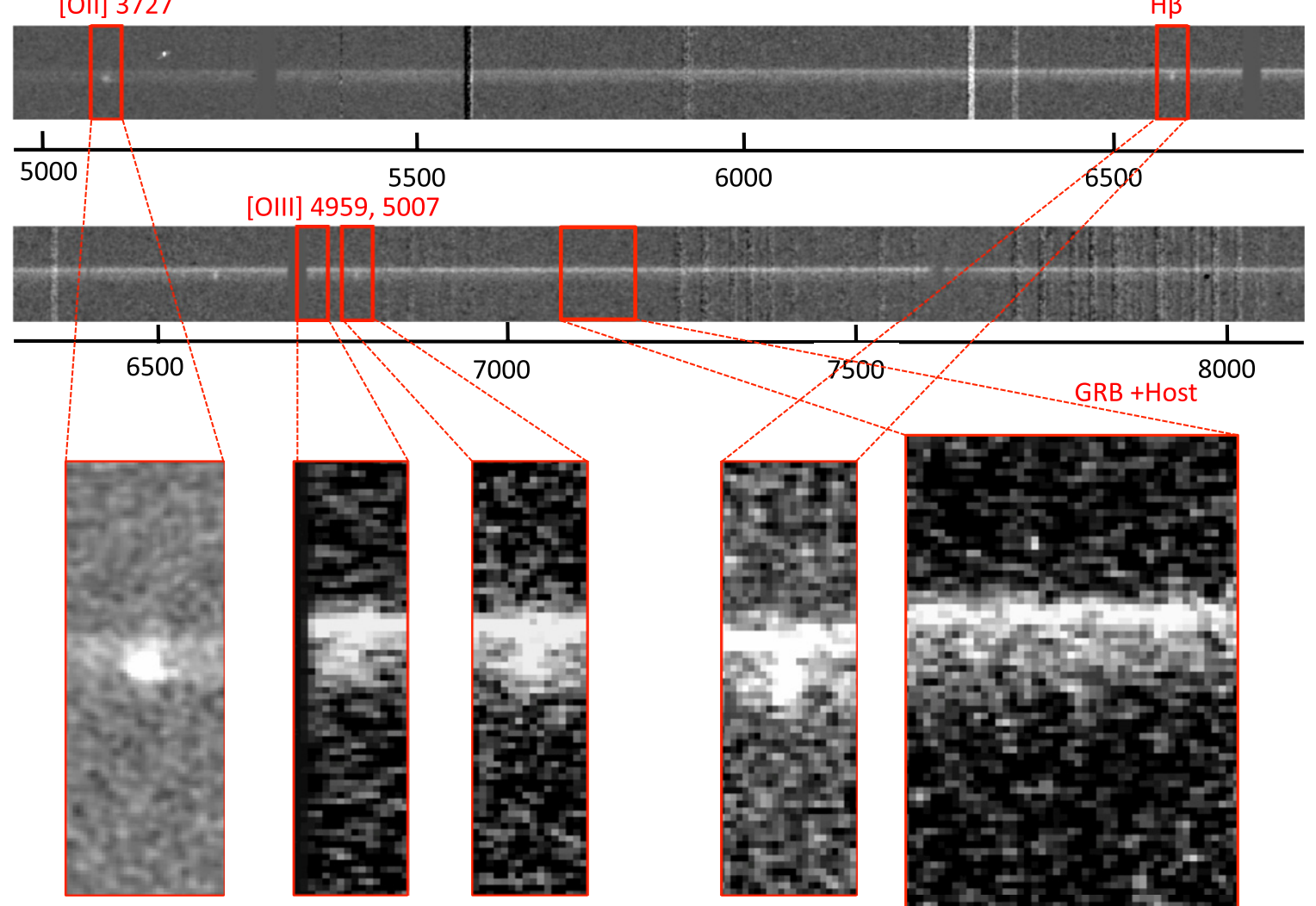

III] 4959, 5007

6000
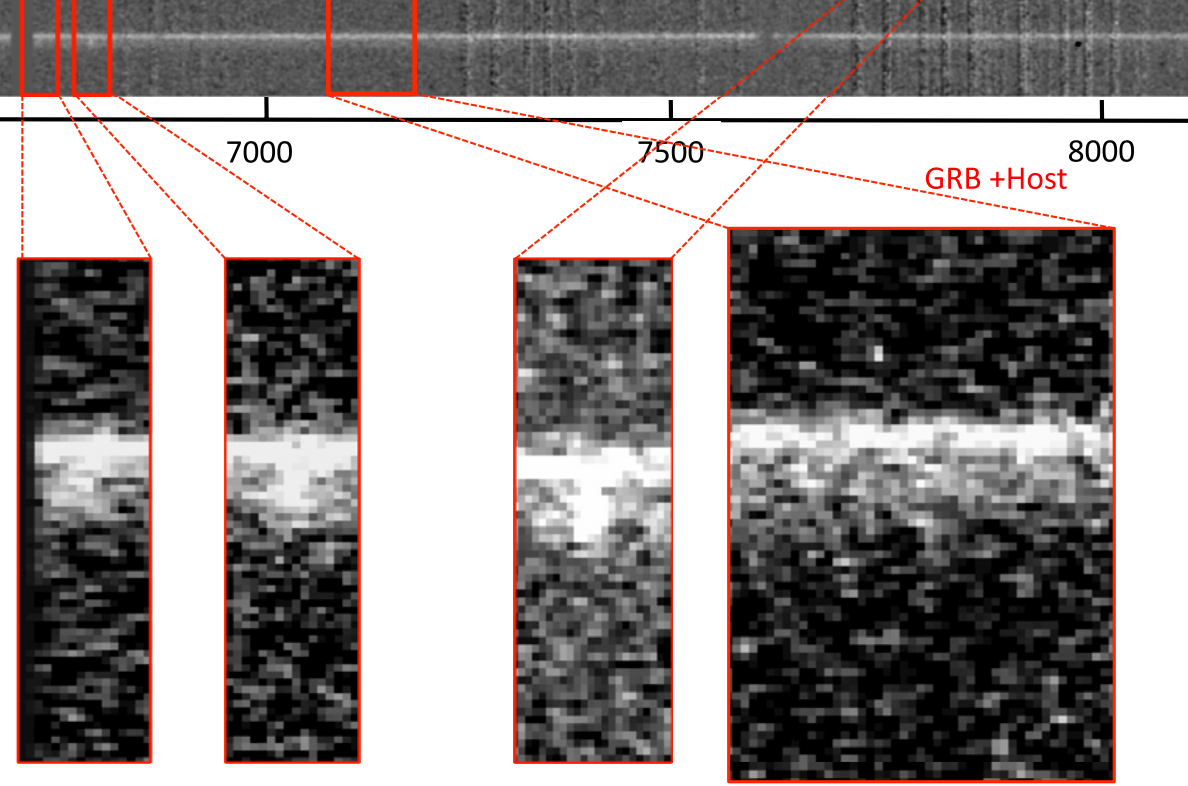

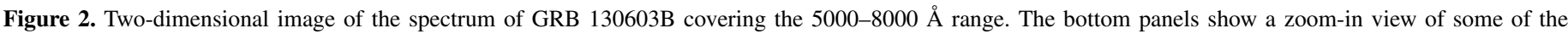

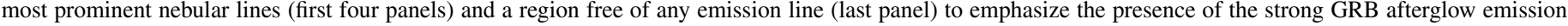
superimposed on the faint host galaxy.

(A color version of this figure is available in the online journal.)

Dokkum 2001). A sky region close in the spatial direction, but unaffected by the spectral trace, was used for sky subtraction. The two-dimensional spectra were then coadded. Figure 2 presents the processed data which reveal the spectrum of the extended galaxy exhibiting a faint continuum and a series of emission lines. Clearly visible superimposed on the galaxy light is an unresolved (spatially) trace that coincides with the expected position of the afterglow based on our imaging data.

Using the IRAF/APALL routine, we extracted a spectrum corresponding to the entire detected trace, therefore including both the galaxy and the GRB afterglow signal. We then extracted a second spectrum with the aperture restricted to the spatial location of the GRB afterglow. Variance spectra were extracted in both cases evaluating sky contribution in two regions unaffected by the galaxy or the GRB light (plotted in gray in Figure 3 and with dash lines in Figure 2).
Flux calibration was performed using an observation of the spectrophotometric standard star Feige 110 taken with the same instrument configuration. An air-to-vacuum correction was applied to the $1 \mathrm{D}$ wavelength solution. Using the measured optical brightness of the GRB+host we also corrected for a small slit-loss $(\lesssim 10 \%)$.

On the following day, starting at $T_{0}+1.3$ days after the burst, we observed again the field with Gemini South obtaining $3 \times 180$ s imaging exposures with the GMOS camera in the $g^{\prime}, r^{\prime}$, $i^{\prime}$ bands and a single $900 \mathrm{~s}$ spectrum with the same configuration used the first night. The afterglow had faded considerably in comparison with the host galaxy. The reduced spectrum revealed no trace from the GRB, but only a faint continuum from the host, with identical emission lines superimposed.

We also obtained an optical spectrum of the host galaxy of GRB 130603B on 2013 June 6 UT with the Deep Imaging 

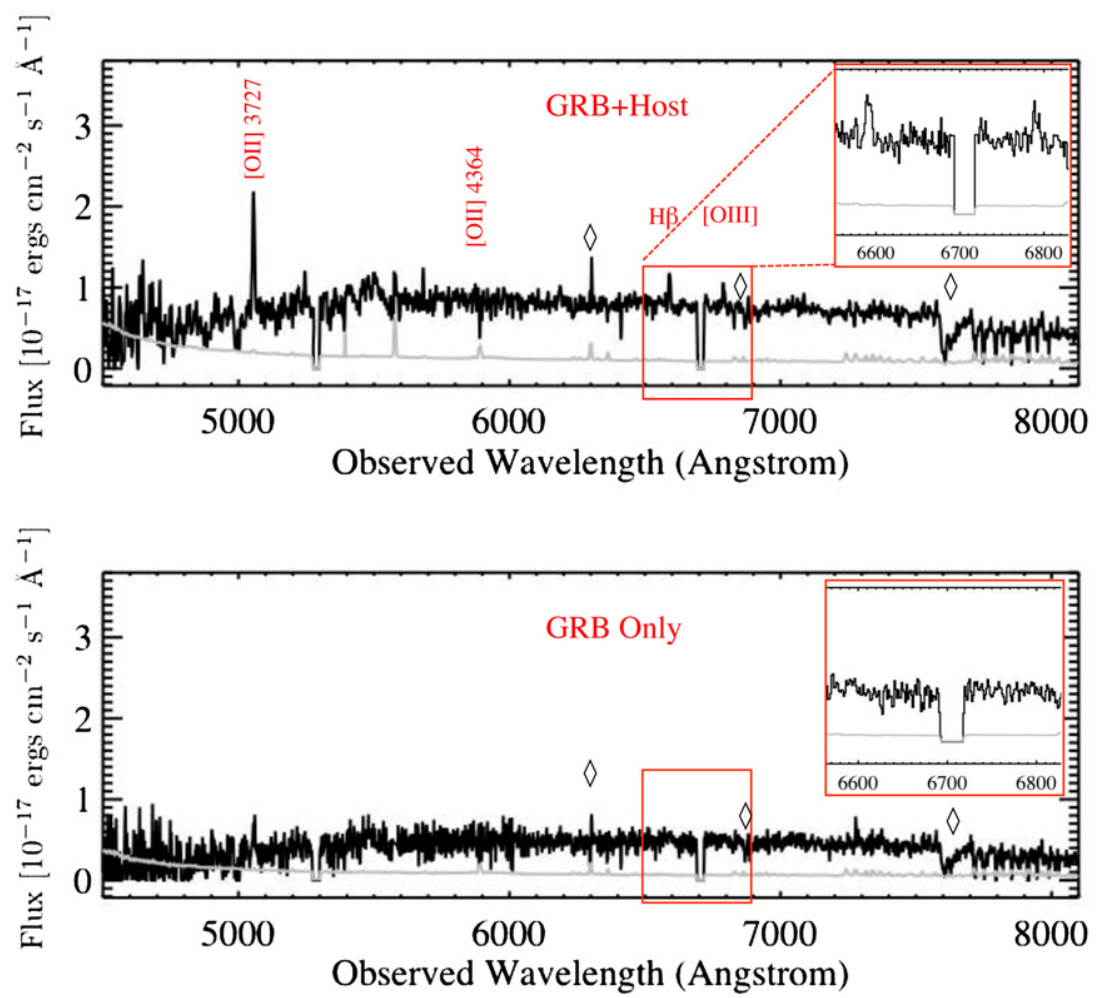

Figure 3. Top: GMOS spectrum of the GRB and the host galaxy using the data obtained the first night (black are the data, gray the variance spectrum, and diamonds indicate the strongest atmospheric telluric lines). Inset shows a zoom-in section at the location of the $\mathrm{H} \beta$ and $[\mathrm{O}$ III] $\lambda \lambda 4959$, 5007 lines. Bottom: same as at the top, but extracting solely the spectrum at the GRB location. In the inset we show a zoom-in section: no emission lines are detected at those locations while very little emission is present at the location of $[\mathrm{O} \mathrm{III}] \lambda 3727$, indicating the GRB explosion location is not a region of especially active star-formation.

(A color version of this figure is available in the online journal.)

Multi-Object Spectrograph (DEIMOS; Faber et al. 2003) mounted on the $10 \mathrm{~m}$ Keck II telescope. The instrument was configured with the 600 lines $\mathrm{mm}^{-1}$ grating, providing spectral coverage over the region $\lambda=4500-9500 \AA$ with a spectral resolution of $3.5 \AA$. The spectra were optimally extracted (Horne 1986), and the rectification and sky subtraction were performed following the procedure described by Kelson (2003). The slit was oriented at an angle such to include the host nucleus and the GRB location. Flux calibration was performed relative to the spectrophotometric standard star BD+262606.

Finally, on June 16 UT, we imaged the field with the GeminiSouth telescope in $g^{\prime}, r^{\prime}, i^{\prime}$, and $z^{\prime}$ bands to estimate the galaxy contribution at a time when the afterglow was expected to have faded well beyond our detectability.

\section{SPECTRAL ANALYSIS}

Figure 3 presents extractions associated with the combined afterglow and galaxy and for an aperture restricted to the afterglow location. In the combined spectrum, we identify a series of nebular emission lines (e.g., [O II] $\lambda 3727,[\mathrm{O} \mathrm{III]} \lambda \lambda 44959,5007$, and $\mathrm{H} \beta$ ), at a common redshift $z=0.3568 \pm 0.0005$. We associate these lines to $\mathrm{H}$ II regions near the center of the galaxy, spatially offset from the afterglow location (Figure 2). The only significant absorption features present in the spectrum are $\mathrm{Ca}$ II $\mathrm{H}+\mathrm{K}$ features, despite the fact that the flux and signal-to-noise ratio $(\mathrm{S} / \mathrm{N})$ are lower (see Section 4.3)

The narrow aperture centered on the afterglow location shows a largely featureless continuum (lower panel of Figure 3). At wavelengths $\lambda \gtrsim 6000 \AA$ the two-dimensional spectrum and our imaging indicate the afterglow dominates the flux. We measure a spectral slope in the optical of $v f_{v} \propto v^{-\beta_{o}}$ with $\beta_{o}=0.62 \pm 0.17$.

Examining the GRB afterglow spectrum at the wavelengths of the galaxy's nebular lines we detect little or no emission (see zoom-in regions in Figure 3). Upper limits on the line fluxes are given in Table 1.

Figure 4 shows a section of the two-dimensional spectrum centered on the location of the Ca II doublet (dashed lines represent the variance spectrum). In the outset we highlight this region in the corresponding extracted spectra for the afterglow only and the afterglow+host: at the location of the redshifted Ca II H\&K we see a decrement in flux in the two-dimensional spectrum and in both extracted spectra. Using the variance spectra we can determine the pixel-by-pixel flux error. At the expected location we detect a redshifted Ca II K-line at $\sim 4 \sigma$ significance level in the afterglow+galaxy spectrum $(2.5 \sigma$ in the afterglow only), while the Ca II H-line only at $2 \sigma$ (and $\lesssim 1 \sigma$ ). This result, in combination with the emission lines detected, places strong constraints on the GRB redshift $(z \gtrsim 0.3568)$ and suggests a likely association of the GRB with the galaxy.

\section{RESULTS}

\subsection{Properties of the GRB and its Afterglow}

Our second and third imaging epochs do not show any clear sign of the afterglow either in the single exposures nor in coadded ones. Assuming that the afterglow completely faded below our detection limit at $T_{0}+1.3$ days we subtract from our first epoch of imaging this "reference" to measure the afterglow flux at the time of our initial observation. Using the 


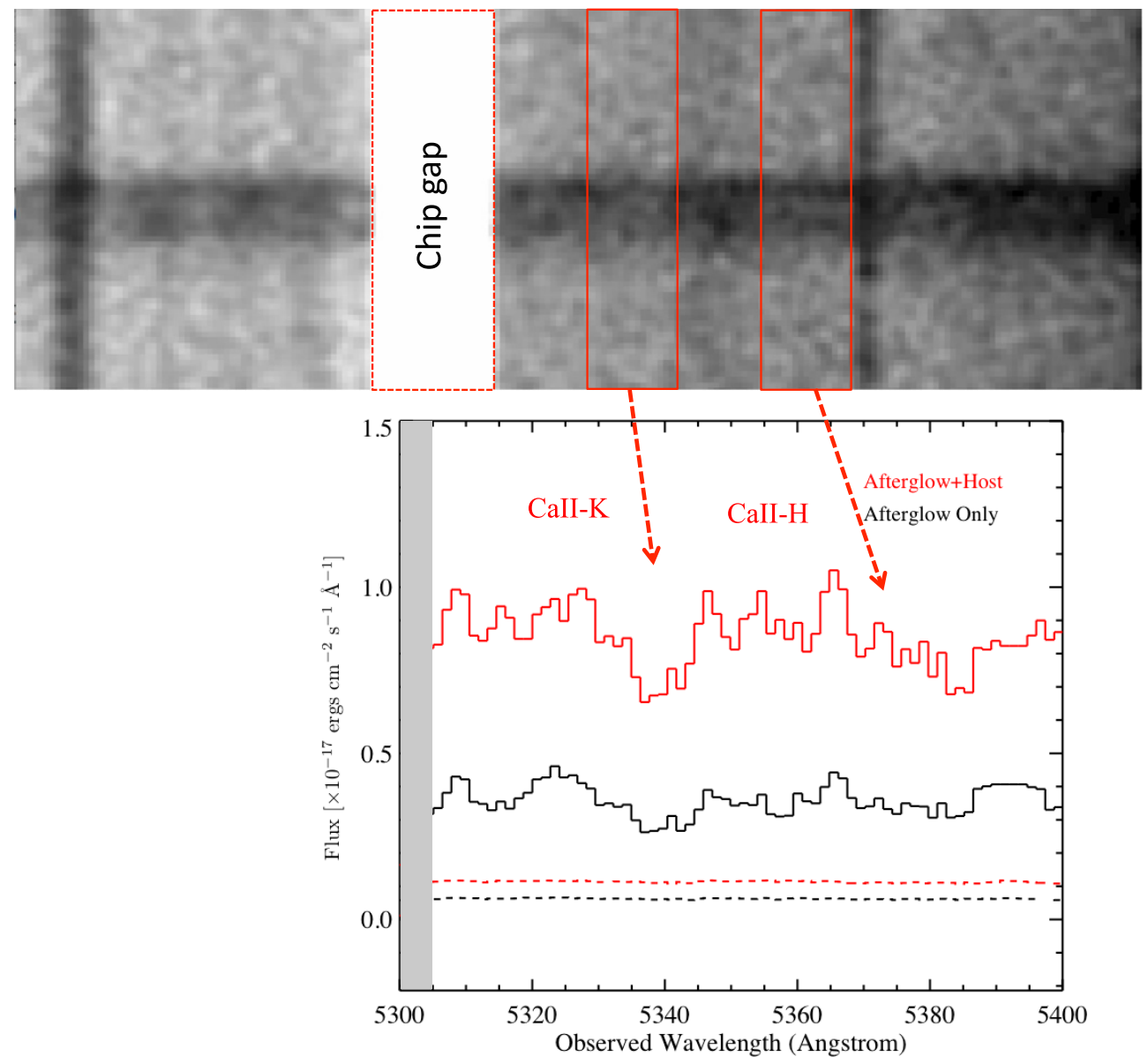

Figure 4. Section of the two-dimensional spectrum centered on the Ca II H\&K absorption features, re-binned in order to enhance the color contrast (top panel). The afterglow and host traces are well visible and at the position of the Ca II lines (in particular the K line at $\lambda_{\text {rest }}=3933.7 \AA$ ) there is a decrement in the continuum flux. In the bottom panel we present the same region for the extracted one-dimensional spectra (black and red for the Afterglow only or the Afterglow+Host galaxy traces respectively, while in dash we plot the sigma spectra). Also in this case, despite the low $\mathrm{S} / \mathrm{N}$, the absorption features are evident and allow, in combination with the emission lines (see Figure 2) to secure the redshift of the GRB at $z=0.3568 \pm 0.0005$.

(A color version of this figure is available in the online journal.)

HOTPANTS ${ }^{10}$ code and nearby point sources from SDSS for calibration, we measure the following, extinction corrected, AB magnitudes: $g^{\prime}=22.09( \pm 0.04), r^{\prime}=21.52( \pm 0.05)$, and $i^{\prime}=21.18( \pm 0.11)$. We derive, at $T_{0}+0.35$ days, an afterglow brightness of $r^{\prime}=21.52 \pm 0.05$, after correcting for Galactic extinction $(E(B-V)=0.02)$ : this value is similar to other optically-detected short GRBs observed around the same epoch (Nicuesa Guelbenzu et al. 2012; Kann 2013; Kann et al. 2011; Berger 2010). Moreover, using the data from June 16 as a "reference" does not change these results, while a similar procedure operated between our second and third epochs, allows us to place stringent upper limits on the afterglow brightness at $T_{0}+1.3$ days $\left(r^{\prime}>25.2\right.$ and $i^{\prime}>24.5$. $)$, confirming our assumption that indeed the optical afterglow was undetectable by Gemini. Interestingly, almost at similar time of our latest Gemini observation, a near-IR counterpart was still visible in the HST data as reported by Berger et al. (2013) and Tanvir et al. (2013).

The peak of the galaxy emission and the centroid of the afterglow emission derived from the subtracted image (see last panel on the right in Figure 1) are separated by $\delta r=0^{\prime} 92 \pm 0.10$, corresponding to a projected distance of $4.8 \mathrm{kpc}( \pm 0.5)$ at

\footnotetext{
${ }^{10}$ See http://www.astro.washington.edu/users/becker/hotpants.html.
}

$z=0.3568$. At this redshift the probability of a random association between the galaxy and the GRB at such distance $\delta r$ is very small $\left(P(<\delta r)<10^{-3}\right.$; see also Bloom et al. 2002).

Based on our $g^{\prime}, r^{\prime}, i^{\prime}$ photometry we derive an optical spectral index $\beta_{o}=0.54 \pm 0.12$ (assuming $v F_{o, v} \propto v^{-\beta_{o}}$ ) consistent with analysis of our optical spectrum and similar to other SGRBs (Nicuesa Guelbenzu et al. 2012).

\subsection{Properties of the Host Galaxy}

At $z=0.3568$ the $r^{\prime}$ band magnitude samples the rest-frame $B$-band luminosity of the host. Therefore, using our second epoch observations we derive $r^{\prime}=20.76 \pm 0.06 \mathrm{mag}$ for the GRB host and we estimate a rest-frame, $k$-corrected, absolute $B$-band magnitude (AB) of $M_{B}=-20.96$. Despite the brightness of this galaxy ( $\sim L^{*}$; Zucca et al. 2009), the derived luminosity is not unusual among the short-GRB hosts population (Figure 5; Berger 2009). Using the late-time multiband photometry and the IDL package kcorrect (Blanton \& Roweis 2007) in a similar fashion to Werk et al. (2012) we estimate the mass of the host galaxy as $\mathcal{M} \approx 5.0 \times 10^{9} M_{\odot}$.

The GMOS spectra from the first two nights and the DEIMOS data cover key nebular emission lines. We measured the fluxes of these lines using the latter data, but for the ones also detected in the GMOS spectra we obtain similar results (Table 1). We 
Table 1

Properties of the Host and Afterglow

\begin{tabular}{|c|c|}
\hline & Value \\
\hline$\alpha_{\text {Host }}$ & $11: 28: 48.227$ \\
\hline$\delta_{\text {Host }}$ & $+17: 04: 18.42$ \\
\hline$\alpha_{O T}$ & $11: 28: 48.168$ \\
\hline$\delta_{O T}$ & $+17: 04: 18.06$ \\
\hline$z$ & $0.3568 \pm 0.0005$ \\
\hline Separation $^{\mathrm{a}}(\delta r)$ & $0{ }^{\prime \prime} 85 \mathrm{~W}, 00^{\prime \prime} 36 \mathrm{~S}$ \\
\hline $\mathrm{P}(\leqslant \delta r)$ & $0.00064^{\mathrm{b}}$ \\
\hline$F_{[\mathrm{O} \text { І }] \lambda 3727}$ & $21.5( \pm 0.4)^{\mathrm{c}}$ \\
\hline$F_{\mathrm{GRB},[\mathrm{OII}]} \lambda 3727^{\mathrm{d}}$ & $\leqslant 5.8^{\mathrm{c}}$ \\
\hline$F_{\mathrm{H} \beta}$ & $9.36( \pm 0.3)^{\mathrm{c}}$ \\
\hline$F_{\mathrm{GRB}, \mathrm{H} \beta^{\mathrm{d}}}$ & $\leqslant 1.44^{\mathrm{c}}$ \\
\hline$F_{[\mathrm{O}}$ III] $\lambda 4960$ & $1.47( \pm 0.12)^{\mathrm{c}}$ \\
\hline 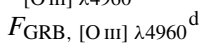 & $\leqslant 1.45^{\mathrm{c}}$ \\
\hline$F_{[\mathrm{O} \text { III }] \lambda 5008}$ & $6.19( \pm 0.23)^{\mathrm{c}}$ \\
\hline 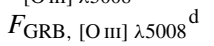 & $\leqslant 2.31^{\mathrm{c}}$ \\
\hline$F_{\mathrm{H} \alpha}$ & $40.2( \pm 0.2)^{\mathrm{c}}$ \\
\hline$F_{\mathrm{N}} \mathrm{II} \lambda 6584$ & $10.4( \pm 0.3)^{\mathrm{c}}$ \\
\hline$M_{\text {Host,B }}$ & $-20.96( \pm 0.07)^{\mathrm{e}}$ \\
\hline $\mathrm{SFR}_{\text {Host }}$ & $1.84 M_{\odot} \mathrm{yr}^{-1}$ \\
\hline $12+\log (\mathrm{O} / \mathrm{H})$ & $8.7 \pm 0.2$ \\
\hline $\mathcal{M}$ & $5 \times 10^{9} M_{\odot}$ \\
\hline$A_{V}$ & $1.3 \mathrm{mag}$ \\
\hline
\end{tabular}

Notes. Summary of the Afterglow and Host galaxy properties as derived from our photometric and spectroscopic observations. The nebular lines fluxes reported here are the raw values as measured from our spectra (see text for the final values and derived quantities). ${ }^{\text {a }} 0$ '.92 ( \pm 0.10$)$ projected or $4.8 \pm 0.5 \mathrm{kpc}$.

${ }^{\mathrm{b}}$ Probability to find such a host galaxy at projected distance $\leqslant \delta r$.

${ }^{\mathrm{c}}$ In units of $10^{-17} \mathrm{erg} \mathrm{cm}^{-2} \mathrm{~s}^{-1}$.

${ }^{\mathrm{d}}$ Upper limit on the galaxy line-flux measured from the GRB afterglow spectrum.

${ }^{\mathrm{e}}$ Rest-frame $B$ magnitude derived from observed $r$.

apply standard emission-line analysis to derive intrinsic galaxy properties. We have estimated the optical extinction using the Balmer lines decrement and assuming case-B recombination Calzetti et al. (1994), Kennicutt (1998): we derived $E(B-V)=$ $0.43\left(A_{V}=1.3 \mathrm{mag}\right.$, assuming a Milky-Way extinction curve). Using the calibration of (Kennicut 1998), the [O II] line luminosity gives a $\mathrm{SFR}([\mathrm{O} \mathrm{II}])=1.7 M_{\odot} \mathrm{yr}^{-1}$. Similarly, from the $\mathrm{H} \alpha$ luminosity we derive $\operatorname{SFR}(\mathrm{H} \alpha)=1.84 M_{\odot} \mathrm{yr}^{-1}$. Together with the $B$-band luminosity, we derive a specific SFR: $\mathrm{sSFR}=\mathrm{SFR} / L_{B}^{*} \gtrsim 2.1 M_{\odot} \mathrm{yr}^{-1} L^{*-1}$. As in other many short GRB hosts, this value is consistent with a star-forming galaxy (see Figure 5; Berger 2009).

Also, collisionally-excited oxygen and the $\mathrm{H} \alpha$ and $\mathrm{H} \beta$ Balmer series recombination lines provide an estimate of the gasphase oxygen abundances of the host galaxy. We adopt the $R_{23}=\left(F_{[\mathrm{OII}] \lambda 3727}+F_{[\mathrm{O} \text { III }] \lambda \lambda 4959,5007}\right) / F_{\mathrm{H} \beta}$ metallicity indicator (Kobulnicky \& Kewley 2004; Pagel et al. 1979), which depends on both the metallicity and the ionization state of the gas. To help disentangle a degeneracy in the values, we use the $O_{32}$ indicator $\left(O_{32}=F_{[\mathrm{O} \text { III }] \lambda \lambda 4959,5007} / F_{[\mathrm{O} \text { II] } \lambda 3727}\right)$.

However, our measured fluxes still allow for two solutions: $\approx 8.7$ for the upper $R_{23}$ branch and $\approx 7.8-8.3$ for the lower branch. The typical error in this measurement, due to systematic uncertainty in the calibration of these metallicity relations is typically $\sim 0.2 \mathrm{dex}$. No field galaxy at $z \sim 0.4$ with a similar brightness to GRB 130603B exhibits metallicities consistent with the lower-branch of our $R_{23}$ analysis. Furthermore, based on the detection of $\mathrm{H} \alpha$ and $\mathrm{N}_{\text {II }} \lambda 6583$ lines in the DEIMOS
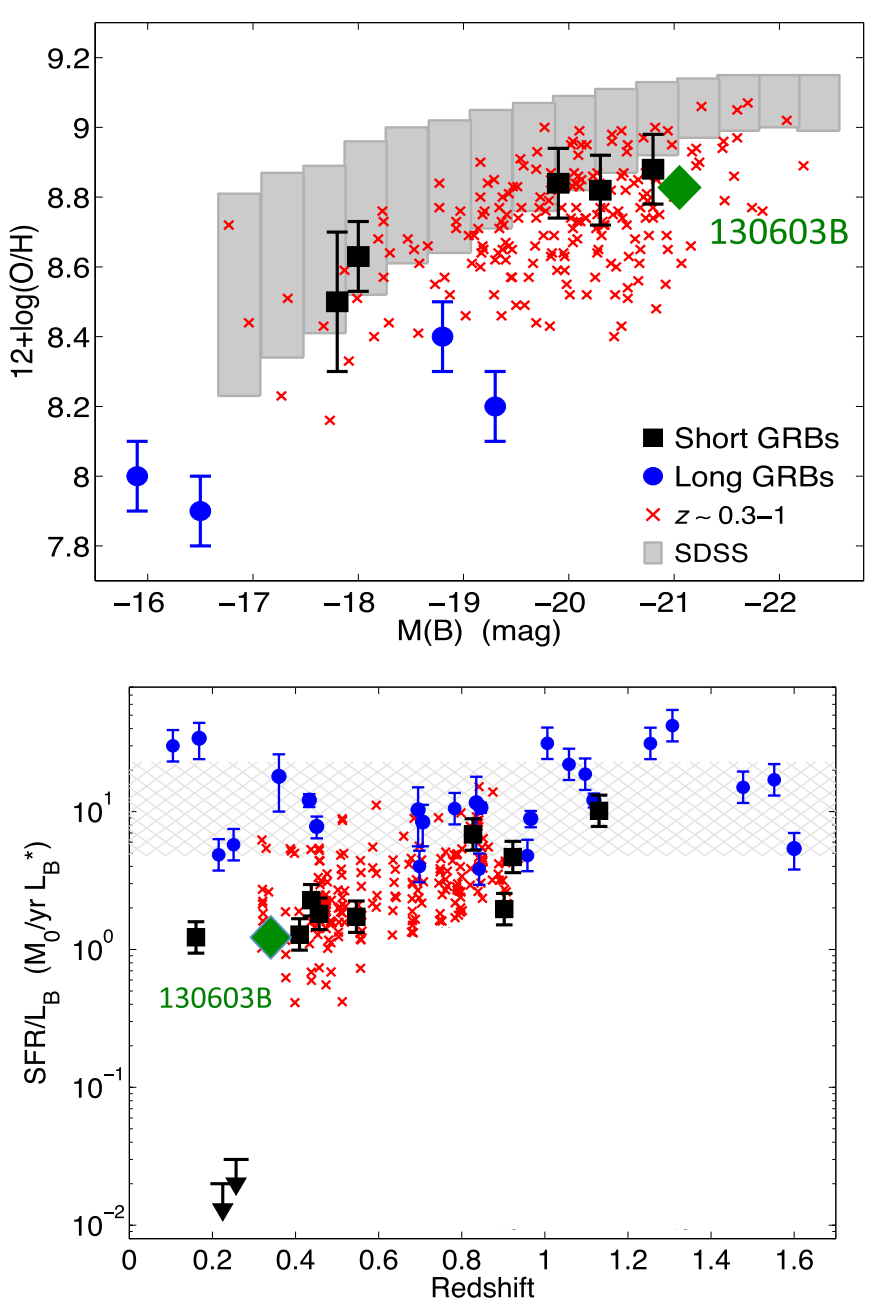

Figure 5. Adapted from Berger 2010. Top: metallicity as function of host galaxy absolute B-magnitude of long (blue points) and short (black squares). Crosses are field galaxies from Kobulnicky \& Kewley (2004), while gray bars are SDSS galaxy at $z \sim 0.1$. GRB 130603B is indicated with a green diamond and clearly shows similar property of the short GRBs host population. Bottom: specific star formation rate vs. redshift for long (blue), short (black) GRB hosts as well as field galaxies (red crosses). Again the green diamond marks the short GRB 130603B. It is evident that the host of GRB 130603B is very similar to other SGRB hosts, though the properties of the GRB explosion site could be very different from its host center.

(A color version of this figure is available in the online journal.)

spectrum we place a lower limit on the metallicity of $12+$ $\log (\mathrm{O} / \mathrm{H}) \gtrsim 8.5$. So we can conclude the galaxy has approximately solar metallicity (Asplund et al. 2005).

\subsection{The Afterglow Spectrum of GRB 130603B}

GRB 130603B marks one of the best cases in which, for a short GRB, an afterglow-dominated spectrum has been obtained. Overall, the extracted GRB afterglow spectrum presents a smooth, almost featureless continuum (Figure 3 lower panels). Despite the absence of strong spectral features, we can still constrain the GRB redshift from these data alone: (1) the lack of a $\operatorname{Ly} \alpha$ forest requires that the GRB exploded at $z_{\mathrm{GRB}}<2.9$; (2) the appearance of weak $\mathrm{Ca}$ II $\mathrm{H}+\mathrm{K}$ absorption lines (see Figure 4) as also seen by Thoene et al. (2013) and SánchezRamírez et al. (2013), gives a strong indication that the GRB redshift is at least a the emission line redshift $\left(z_{\mathrm{GRB}} \gtrsim 0.3586\right)$; (3) if one assumes the afterglow spectrum will exhibit significant ( $>0.3 \AA$ ) $\mathrm{Mg}$ II absorption from the surrounding interstellar or 
circumgalactic medium, then we set an upper limit $z_{\mathrm{GRB}}<0.78$ based on its non-detection. All of these constraints are consistent with GRB 130603B having occurred within the coincident galaxy. Ultimately, an indisputable measurement of $z_{\mathrm{GRB}}$ from an afterglow spectrum may require the detection of finestructure transitions excited by the GRB itself (Prochaska et al. 2005) and/or the observed termination of the Ly $\alpha$ forest. Unfortunately, our wavelength coverage and the small number of this kind of features that would have been redshifted in our observed window (and therefore detected) prevent us from obtaining such a secure measurement. Furthermore, analysis of these transitions would reveal physical properties of the progenitor environment (e.g., metallicity, size) and may offer new insight into the progenitors of SGRBs.

Associating GRB 130603B to the neighboring galaxy, we may place an upper limit to the SFR at the position of the event. No strong emission lines are identified, in contrast to the putative host spectrum: the striking difference can be seen at the location of the $\mathrm{H} \beta$ and [O III] $\lambda \lambda 4959,5007$ lines (insets in Figure 3). This indicates that GRB 130603B, unlike the majority of long-duration GRBs, exploded in a region of very minimal, if not negligible, star-formation. Based on the integrated flux at the location of the $\mathrm{H} \beta$ line, we place an upper limit $\mathrm{SFR}(\mathrm{H} \beta) \lesssim 0.4 M_{\odot} \mathrm{yr}^{-1}$. This provides further support for models unassociated with massive star formation.

\section{SUMMARY AND CONCLUSIONS}

We present our Gemini rapid follow-up campaign on the afterglow of the short GRB 130603B. Despite the intrinsic faintness of these events, the low-energy emission of GRB 130603B, in particular in the optical afterglow, was still detectable several hours after the explosion. We triggered our approved ToO program at the Gemini South telescope and obtained a series of images of the GRB field as well as spectroscopic observations with the GMOS camera starting $8 \mathrm{hr}$ after the burst. We repeated similar observations the following night.

Based on our absorption lines analysis and the very small probability of a random association with such a bright galaxy $(P(\leqslant \delta r)=0.00064)$ we conclude that this is the host of GRB 130603B. Our optical images provide firm evidence that this event occurred in the outskirt of a star-forming galaxy $\left(M_{B}=-20.96\right)$, around $4.8 \mathrm{kpc}$ from its center (at the GRB redshift of $z_{\text {grb }}=0.3568$ ). While this offset is not unusual for long duration GRBs (e.g., Bloom et al. 2002), the lack of blue light at the location is very unlike long-duration GRB locations (Fruchter et al. 2006). Deep HST observations also confirm this findings (Berger et al. 2013; Tanvir et al. 2013).

The early-time spectra present a bright afterglow continuum superimposed upon a fainter galaxy. This represents one of the few cases where an afterglow-dominated spectrum for a short GRB has been recorded. The host is an $\sim L^{*}$ galaxy, and the spectrum shows strong nebular emission lines as well as recombination features. We measure a dust-corrected $\mathrm{SFR}=1.84 M_{\odot} \mathrm{yr}^{-1}$ and solar metallicity.

Nevertheless, we were able to extract the GRB spectrum and constrain the properties of the host galaxy at its location: the GRB spectrum present a smooth continuum showing almost no sign of emission lines throughout the wavelength coverage. Also, at the same redshift we identify Ca II absorption lines, which place a strong constraint to the GRB redshift, despite the lack of fine-structure transitions. Therefore, at the GRB location, the star-formation activity is almost negligible $(\operatorname{SFR}(\mathrm{H} \beta) \lesssim$ $\left.0.4 M_{\odot} \mathrm{yr}^{-1}\right)$.
This last result, once more, emphasizes the importance of rapid follow-up observations with large aperture facilities in order to firmly identify the faint afterglows of these shortlived events. Only with a larger sample of data similar to those presented in this work we will be able not only to identify a larger number of short GRBs and measure their redshifts, but also to characterize, via absorption spectroscopy, their explosion site environment and move forward in our understanding of the nature of their progenitors.

A.C. thanks the anonymous referee for the valuable comments and suggestions which have helped significantly to improve the manuscript. A.C., also, thanks D. A. Kann and S. Savaglio for the valuable discussions and comments. Gemini results are based on observations obtained at the Gemini Observatory, which is operated by the Association of Universities for Research in Astronomy, Inc., under a cooperative agreement with the NSF on behalf of the Gemini partnership: the National Science Foundation (United States), the National Research Council (Canada), CONICYT (Chile), the Australian Research Council (Australia), Ministério da Ciência, Tecnologia e Inovação (Brazil), and Ministerio de Ciencia, Tecnología e Innovación Productiva (Argentina). Partial support for this work was provided by NASA through Hubble Fellowship grant HST-HF-51296.01-A awarded by the Space Telescope Science Institute, which is operated by the Association of Universities for Research in Astronomy, Inc., for NASA, under contract NAS 5-26555.

\section{REFERENCES}

Ahn, C. P., Alexandroff, R., Allende Prieto, C., et al. 2012, ApJS, 203, 21 Antonelli, L. A., D’Avanzo, P., Perna, R., et al. 2009, A\&A, 507, L45 Asplund, M., Grevesse, N., \& Sauval, A. J. 2005, in ASP Conf. Ser. 336, Cosmic Abundances as Records of Stellar Evolution and Nucleosynthesis, ed. T. G. Barnes, III \& F. N. Bash (San Francisco, CA: ASP), 25

Barthelmy, S. D., Barbier, L. M., Cummings, J. R., et al. 2005, SSRv, 120, 143 Berger, E. 2007, ApJ, 670, 1254

Berger, E. 2009, ApJ, 690, 231

Berger, E. 2010, ApJ, 722, 1946

Berger, E., Fong, W., \& Chornock, R. 2013, ApJL, 774, L23

Berger, E., Penprase, B. E., Cenko, S. B., et al. 2006, ApJ, 642, 979

Blanton, M. R., \& Roweis, S. 2007, AJ, 133, 734

Bloom, J. S., Kulkarni, S. R., \& Djorgovski, S. G. 2002, AJ, 123, 1111

Bloom, J. S., Prochaska, J. X., Pooley, D., et al. 2006, ApJ, 638, 354

Bromberg, O., Nakar, E., Piran, T., \& Sari, R. 2013, ApJ, 764, 179

Burrows, D. N., Hill, J. E., Nousek, J. A., et al. 2005, SSRv, 120, 165

Calzetti, D., Kinney, A. L., \& Storchi-Bergmann, T. 1994, ApJ, 429, 582

Chen, H.-W., Prochaska, J. X., Bloom, J. S., \& Thompson, I. B. 2005, ApJL, 634, L25

Cucchiara, A., Levan, A. J., Fox, D. B., et al. 2011, ApJ, 736, 7

Cucchiara, A., Perley, D. A., \& Cenko, S. B. 2013, GCN, 14748, 1 de Pasquale, M., \& Melandri, A. 2013, GCN, 14759, 1

Evans, P. A., Goad, M. R., \& Osborne, J. P. 2013, GCN, 14739, 1

Faber, S. M., Phillips, A. C., Kibrick, R. I., et al. 2003, Proc. SPIE, 4841, 1657

Foley, R. J., Chornock, R., Fong, W., Berger, E., \& Jha, S. 2013, GCN, 14745,1

Fong, W., Berger, E., Chornock, R., et al. 2013a, ApJ, 769, 56

Fong, W., Zauderer, B. A., \& Berger, E. 2013b, GCN, 14751, 1

Fong, W.-f., \& Berger, E. 2013, arXiv:1307.0819

Fox, D. B., Frail, D. A., Price, P. A., et al. 2005, Natur, 437, 845

Fruchter, A. S., Levan, A. J., Strolger, L., et al. 2006, Natur, 441, 463

Gehrels, N., Chincarini, G., Giommi, P., et al. 2004, ApJ, 611, 1005

Gorosabel, J., Castro-Tirado, A. J., Tanvir, N., et al. 2010, GCN, 11125, 1

Hjorth, J., Sollerman, J., Gorosabel, J., et al. 2005, ApJL, 630, L117

Hook, I. M., Jørgensen, I., Allington-Smith, J. R., et al. 2004, PASP, 116, 425

Horne, K. 1986, PASP, 98, 609

Jakobsson, P., Hjorth, J., Fynbo, J. P. U., et al. 2004, ApJL, 617, L21

Kann, D. A. 2013, EAS, 61, 309

Kann, D. A., Klose, S., Zhang, B., et al. 2011, ApJ, 734, 96 
Kelson, D. D. 2003, PASP, 115, 688

Kennicutt, R. C., Jr. 1998, ARA\&A, 36, 189

Kobulnicky, H. A., \& Kewley, L. J. 2004, ApJ, 617, 240

Kouveliotou, C., Meegan, C. A., Fishman, G. J., et al. 1993, ApJL, 413, L101

Lee, W. H., Ramirez-Ruiz, E., \& Granot, J. 2005, ApJL, 630, L165

Lee, W. H., Ramirez-Ruiz, E., \& van de Ven, G. 2010, ApJ, 720, 953

Levan, A. J., Tanvir, N. R., Wiersema, K., \& Hartoog, O. 2013, GCN, 14742

Levesque, E. M. 2013, arXiv:1302.4741

Levesque, E. M., Bloom, J. S., Butler, N. R., et al. 2010, MNRAS, 401, 963

Li, L.-X., \& Paczyński, B. 1998, ApJL, 507, L59

Mazets, E. P., Golenetskii, S. V., Ilyinskii, V. N., et al. 1981, Ap\&SS, 80, 119

Melandri, A., Baumgartner, W. H., Burrows, D. N., \& Cummings, J. R. 2013, GCN, 14735

Metzger, B. D., \& Berger, E. 2012, ApJ, 746, 48

Metzger, M. R., Djorgovski, S. G., Kulkarni, S. R., et al. 1997, Natur, 387, 879

Nakar, E. 2007, PhR, 442, 166

Nicuesa Guelbenzu, A., Klose, S., et al. 2012, A\&A, 548, A101

Norris, J., Ukwatta, T. N., Barthelmy, S. D., et al. 2010, GCN, 11113, 1

Norris, J. P., Cline, T. L., Desai, U. D., \& Teegarden, B. J. 1984, Natur, 308,434

Norris, J. P., Gehrels, N., \& Barthelmy, S. D. 2013, GCN, 14746, 1

O’Shaughnessy, R., Belczynski, K., \& Kalogera, V. 2008, ApJ, 675, 566

Pagel, B. E. J., Edmunds, M. G., Blackwell, D. E., Chun, M. S., \& Smith, G. 1979, MNRAS, 189, 95
Perley, D. A., Levan, A. J., Tanvir, N. R., et al. 2013, arXiv:1301.5903 Prochaska, J. X., Chen, H., \& Bloom, J. S. 2005, arXiv:astro-ph/0601057 Prochaska, J. X., Chen, H.-W., \& Bloom, J. S. 2006, ApJ, 648, 95

Řípa, J., Mészáros, A., Wigger, C., et al. 2009, A\&A, 498, 399

Roming, P. W. A., Kennedy, T. E., Mason, K. O., et al. 2005, SSRv, 120, 95

Salvaterra, R., Della Valle, M., Campana, S., et al. 2009, Natur, 461, 1258

Sánchez-Ramírez, R., Castro Tirado, A. J., Gorosabel, J., \& Aceituno, J. 2013, GCN, 14747, 1

Soderberg, A. M., Berger, E., Kasliwal, M., et al. 2006, ApJ, 650, 261

Tanvir, N. R., Fox, D. B., Levan, A. J., et al. 2009, Natur, 461, 1254

Tanvir, N. R., Levan, A. J., Fruchter, A. S., et al. 2013, Natur, 500, 547

Tanvir, N. R., Wiersema, K., Fynbo, J. P. U., Levan, A. J., \& Perley, D. 2010, GCN, 11116, 1

Thoene, C. C., de Ugarte Postigo, A., Gorosabel, J., \& Tanvir, N. R. 2013, GCN, 14744,1

van Dokkum, P. 2001, PASP, 113, 1420

Veres, P., Bagoly, Z., Horváth, I., Mészáros, A., \& Balázs, L. G. 2010, ApJ, 725,1955

Vreeswijk, P. M., Ledoux, C., Raassen, A. J. J., et al. 2013, A\&A, 549, A22

Werk, J. K., Prochaska, J. X., Thom, C., et al. 2012, ApJS, 198, 3

$\mathrm{Xu}$, D., de Ugarte Postigo, A., Malesani, D., \& Schulze, S. 2013, GCN, 14757,1

Zhang, B., Zhang, B.-B., Virgili, F. J., et al. 2009, ApJ, 703, 1696

Zucca, E., Bardelli, S., Bolzonella, M., et al. 2009, A\&A, 508, 1217 